\title{
CORRESPONDENCE
}

\section{The need for precise answers for the goals of precision medicine in alcohol dependence to succeed}

Neuropsychopharmacology (2018) 43:1799-1800; https://doi.org/ 10.1038/s41386-018-0112-y

Dear Editor,

We read the study by Mann et al. [1] with great interest. Clinical trials of pharmacotherapies for alcohol use disorder have yielded inconsistent or suboptimal outcomes, but it may be that drinker characteristics moderate pharmacotherapy efficacy. To begin to answer the important clinical question of "which medication will work best for which patient," Mann et al. [1] grouped drinkers by whether they drank for alcohol's positive and/or negative reinforcing qualities. This yielded: 'reward drinkers' (high-reward and low-relief), 'relief drinkers' (low-reward and high-relief), 'all high drinkers' (high-reward and high-relief) and 'all low drinkers' (low-reward and low-relief). The authors sought "to test differential efficacy of naltrexone and acamprosate based on individual differences in neurotransmitter dysfunction" putatively expected across these subgroups. They predicted "individuals with primarily reward drinking tendencies [reward drinkers w/opioidergic dysfunction] would respond better to naltrexone ... whereas individuals with primarily relief drinking tendencies [relief drinkers w/glutamatergic dysfunction] would respond better to acamprosate" [1]. The study takes a novel precision medicine approach within a rigorously designed clinical trial and a mechanistic focus. It offers immediate potential to address longstanding shortcomings in treatment efficacy and efficiency.

We were surprised, however, to find that the reported analyses never directly tested the differential efficacy of naltrexone versus acamprosate in any subgroups as proposed. Instead, the authors compared the effects of each drug (vs. placebo) for each of the reward and relief drinkers relative to the effects of the same drug (vs. placebo) for all low drinkers. The key finding was a 2 (naltrexone vs. placebo) $\times 2$ (reward drinkers vs. all low drinkers) interaction, which indicated the differential efficacy of naltrexone vs. placebo was improved in reward drinkers relative to all low drinkers (means displayed in Fig. 1a). This finding in a welldesigned study such as this one is perhaps itself worth publication. Its clinical implications for precision medicine, however, are limited as it only implies clinicians should choose naltrexone over placebo to treat reward drinkers. It does not speak to whether clinicians should choose naltrexone over acamprosate to treat reward drinkers. The results of their analyses thereby fail to address the proposed research question: "which medication will work best for which patients (i.e. precision medicine)" [1].

To rigorously test the differential efficacy of naltrexone vs. acamprosate for reward drinkers, the authors could have tested the 2 (naltrexone vs. acamprosate) $\times 2$ (reward drinkers vs. all low drinkers) interaction (Fig. 1b). Unfortunately, the observed pattern of means provides no evidence that clinicians should choose naltrexone over acamprosate when they treat reward drinkers specifically. It also does not demonstrate any match between selective drug mechanisms and putative subgroup dysfunction that serves as the foundation for this precision medicine study.

Although this correspondence focuses only on one recent paper, these analytic concerns and related issues remain rampant in clinical research $[2,3]$. As the rigor of research increases through open science practices, such as preregistration of analyses,
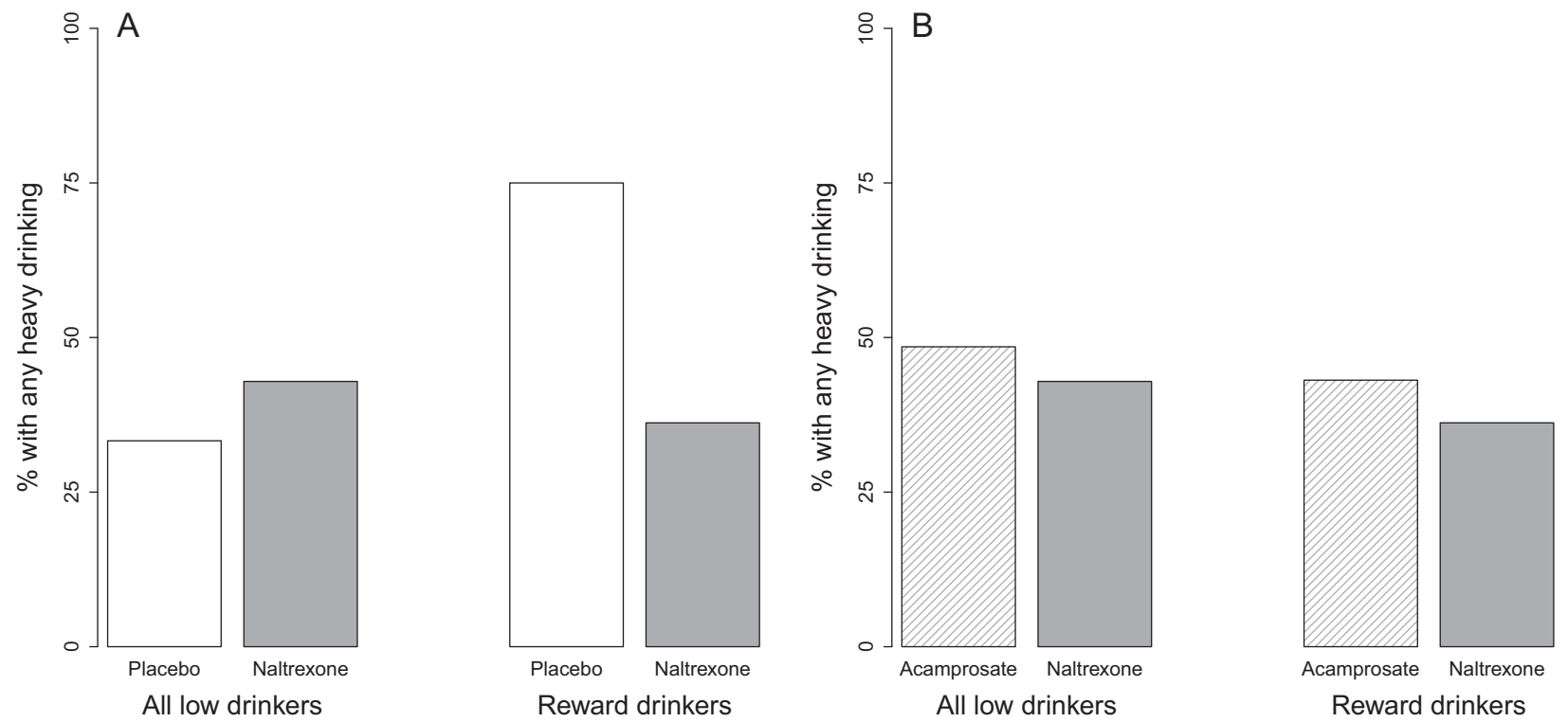

Fig. 1 Means for percent of drinkers with any heavy drinking taken from Table 3 in [1]

Received: 15 February 2018 Accepted: 18 May 2018

Published online: 12 June 2018 
1800

registered reports, increased transparency, and related endeavors [4-6], it will be important to couple carefully the clinically guided hypotheses with appropriate statistical analyses that directly test them.

\section{ADDITIONAL INFORMATION}

Competing interests: The authors declare no competing interests.

Daniel E. Bradford ${ }^{1}$, Gaylen E. Fronk' ${ }^{1}$, Sarah J. Sant'Ana', Katherine P. Magruder ${ }^{1}$, Jesse T. Kaye ${ }^{1}$ and John J. Curtin ${ }^{1}$

${ }^{1}$ Department of Psychology, University of Wisconsin-Madison, 1202W. Johnson St., Madison, WI 53706, USA Correspondence: Daniel E. Bradford (dbradford@wisc.edu)

\section{REFERENCES}

1. Mann K, Roos CR, Hoffmann S, Nakovics H, Leménager T, Heinz A, et al. Precision medicine in alcohol dependence: a controlled trial testing pharmacotherapy response among reward and relief drinking phenotypes. Neuropsychopharmacol Publ Am Coll Neuropsychopharmacol. 2018;43:891-9.

2. Altman DG, Moher D, Schulz KF. Harms of outcome switching in reports of randomised trials: CONSORT perspective. BMJ. 2017;356:j396.

3. Simmons JP, Nelson LD, Simonsohn U. False-positive psychology: undisclosed flexibility in data collection and analysis allows presenting anything as significant. Psychol Sci. 2011;22:1359-66.

4. Lakens D, Adolfi FG, Albers CJ, Anvari F, Apps MAJ, Argamon SE, et al. Justify your alpha. Nat Hum Behav. 2018;2:168-71.

5. Nosek BA, Alter G, Banks GC, Borsboom D, Bowman SD, Breckler SJ, et al. SCIENTIFIC STANDARDS. Promoting an open research culture. Science. 2015;348:1422-5.

6. Simmons JP, Nelson LD, Simonsohn U. A 21 word solution. 2012. https://doi.org/ $10.2139 /$ ssrn.2160588 